\title{
A COMPARISON BETWEEN TESTS FOR CHANGES IN THE ADJUSTMENT COEFFICIENTS IN COINTEGRATED SYSTEMS
}

Marco R. Barassi

University of Birmingham

Guglielmo Maria Caporale

Brunel University, London

Stephen G. Hall

University of Leicester

Follow this and additional works at: https://fordham.bepress.com/crif_seminar_series

Part of the Finance and Financial Management Commons

\section{Recommended Citation}

Barassi, Marco R.; Caporale, Guglielmo Maria; and Hall, Stephen G., "A COMPARISON BETWEEN TESTS FOR CHANGES IN THE ADJUSTMENT COEFFICIENTS IN COINTEGRATED SYSTEMS" (2007). CRIF Seminar series. 4.

https://fordham.bepress.com/crif_seminar_series/4 


\title{
A COMPARISON BETWEEN TESTS FOR CHANGES IN THE ADJUSTMENT COEFFICIENTS IN COINTEGRATED SYSTEMS
}

\author{
Marco R. Barassi \\ University of Birmingham
}

\author{
Guglielmo Maria Caporale \\ Brunel University, London
}

\author{
Stephen G. Hall \\ University of Leicester
}

\begin{abstract}
In this paper we examine several approaches to detecting changes in the adjustment coefficients in cointegrated VARs. We adopt recursive and rolling techniques as mis-specification tests for the detection of non-constancy and the estimation of the breakpoints. We find that inspection of the recursive eigenvalues is not useful to detect a break in the adjustment coefficients, whilst recursive estimation of the coefficients can only indicate non-constancy, but not the exact breakpoint. Rolling estimation is found to perform better in detecting non-constancy in the parameters and their true value after the breakpoint. However, it only detects a region where the break is likely to occur. To overcome the drawbacks of these techniques, we use an OLS-based sequential test. To assess its performance, we derive its critical values for different sample sizes. Monte Carlo evidence shows that the test has reasonably good power even in moderately sized samples and that it can be used as a graphical device, as it shows a kink at the breakpoint. As a benchmark we use the Kalman filter, of which we analyse the performance on the same data generating processes (DGP).
\end{abstract}

Keywords: Adjustment coefficients, Structural Break, Sequential Test, Monte Carlo Methods

JEL classification: C12, C15, C32

Corresponding author: Professor Guglielmo Maria Caporale, Brunel Business School, Brunel University, Uxbridge, Middlesex UB8 3PH, UK. Tel.: +44 (0)1895 266713. Fax: +44 (0)1895 269770. Email: Guglielmo-Maria.Caporale@brunel.ac.uk

Financial support from ESCR grant no. R00222955, Interest Rate Linkages in a Changing World, is gratefully acknowledged. We are also grateful to Fabio Spagnolo, Nicola Spagnolo, Andrea Cipollini and an anonymous referee for useful comments and suggestions. 


\section{$1 \quad$ Introduction}

As a result of phenomena like oil shocks and sudden policy changes, econometric models with constant coefficients have been found to perform relatively poorly both for forecasting and policy analysis purposes. Changes in the coefficients of econometric models have normally described as structural breaks. Such problems have been addressed using models with continuous parameter changes such as the Kalman filter, outlier models (i.e. sudden shocks may produce outliers), switching regression models with abrupt or gradual switches (Markov-Switching, smooth transition models). However, before using any of these approaches it is customary to try to detect whether such structural changes have occurred.

The underlying motivation for this paper is a simple apparent contradiction, which exists in two major branches of the applied econometric literature. On the one hand we have a very large literature which attests to the pervasive presence of structural breaks in the real world - this is not simply the formal econometric literature (Perron 1989, Hansen 1992, Lumsdaine and Papell 1997, Stock and Watson 1993 among many others), but also includes the general world of economics (Lucas 1988) where we see such evident structural breaks as the creation of the European Monetary Union, the unification of Germany or the restructuring of the financial system which happened throughout the 1980's. Contradicting this, however, we find many examples of stable long-run relationships in the form of co-integrating vectors which appear to exist and remain stable despite the presence of such large changes.

The contention of this paper is that these two stylised facts can be reconciled if we recognise that many of the most important structural changes come in the form of a changing causal structure. So major changes may occur while the basic long-run relationships remain stable. A good example of this is the European Monetary Union. There has been a long established relationship between national price levels and currencies which has been widely tested in the context of the purchasing power parity (PPP) hypothesis. Under floating exchange rates it is usually assumed that the exchange 
rate will move to equalise prices between any two countries. When a monetary union is formed this obviously no longer happens, as the exchange rate is irrevocably fixed. However, since PPP does not disappear, the causal structure must change so that the price levels react to each other to remain in line. The long-run relationship does not change, but the way the system works in a causal sense does. In a modern cointegration framework (Johansen 1988, 1991) this is governed by the $\alpha$ matrix. It is structural change in this part of the system which we wish to focus on in this paper.

Within the cointegration framework several tests for structural change have been proposed. Perron (1989) first showed the importance of such tests, arguing that if there is a break in the deterministic trend, unit root tests tend to under-reject the null of a unit root. Taking the breakpoint as exogenous, he suggests a modification of the DickeyFuller test with three different types of deterministic trend functions. These allow, in turn, for a one-off change in the intercept, a change in slope of the trend, and both of them. The null of a unit root is then tested against the alternative of a broken trend stationary. After Perron's (1989) seminal paper several testing methods have been developed where the break point is assumed to be unknown. This is often referred to as an endogenous breakpoint. These procedures comprise recursive (using sub-samples), rolling (using a fixed-size window that moves along the sample), and sequential methods (including switching dummies in the full sample).

Zivot and Andrews (1992) use a sequential unit root test, derive its distribution, and tabulate its critical values. Banerjee, Lumsdaine and Stock (1992) use various recursive and sequential tests which endogenise the breakpoint. They consider the recursive maximum and minimum DF test and the difference between them deriving the asymptotic distribution of the recursive and sequential test statistics and tabulating the relative critical values. Lumsdaine and Papell (1997) extend the analysis to the case of multiple breaks with unknown breakpoints.

This set of procedures for unit root tests has been extended to the tests for cointegration. Here we need to distinguish between breaks in the relationships and breaks in the 
individual variables. Gregory, Nason and Watt (1996) study the sensitivity of the ADF test for cointegration in the presence of a single permanent break. Using Monte Carlo methods they show that the presence of a break results in under-rejection of the null of no cointegration implying the inappropriateness of constant parameter cointegration analysis in such cases. A simple diagnostic test for structural change is suggested by Hao and Inder (1996), who extend the CUSUM test to the case of non-stationary regressors considering the FM-OLS residuals and replacing the error variance with the long-run variance estimate. They derive the asymptotic distribution of the FM-OLS based CUSUM test statistics and tabulate the critical values.

Hansen (1992) derives the asymptotic distribution of a LM test for parameter instability against several alternatives in the context of cointegrated regression models. Quintos and Phillips (1993) develop a test for the null of parameter constancy in cointegrated regressions against the alternative that the coefficients follow a random walk. Gregory and Hansen (1996) propose several tests for the null of no cointegration against the alternative of cointegration in the presence of a possible break in the intercept or the slope coefficients in the cointegrating relation at an unknown point in time.

All the testing procedures considered so far are performed within a FM-OLS environment and are therefore suitable for FM models or single equations. In cointegrated systems a la Johansen $(1988,1991)$ we can observe four types of structural changes of which three occur in the long-run structure of the model. To explain this let

$$
\Delta X_{t}=\Gamma_{1} \Delta X_{t-1}+\ldots+\Gamma_{k-1} \Delta X_{t-k+1}+\alpha \beta^{\prime} X_{t-k}+\varepsilon_{t}
$$

be the usual cointegrated model in error correction form where $X_{t}$ is a p-dimensional matrix of non-stationary $\mathrm{I}(1)$ variables, $\Gamma_{\mathrm{i}}$ is the matrix of short-run parameters, the error term $\varepsilon_{t} \sim$ iidN $(0, \Sigma), \beta$ is the cointegrating matrix and $\alpha$ is the matrix of adjustment coefficients that determines the long-run causal structure, and $\alpha \beta^{\prime}=\Pi$ has reduced rank $r$ $<p$. It is convenient to rewrite the model as 


$$
Z_{0 t}=\Theta Z_{1 t}+\alpha \beta^{\prime} Z_{2 t}+\varepsilon_{t}
$$

where $\Theta=\left(\Gamma_{1}, \ldots, \Gamma_{k-1}\right), \quad Z_{0 t}=\Delta X_{t}, \quad Z_{1 t}=\left(\Delta X_{t-1}^{\prime}, \ldots, \Delta X_{t-k+1}^{\prime}\right)^{\prime}, \quad Z_{2 t}=X_{t-k}$. Using this parameterisation we can see, first of all, that changes may occur over time in the shortrun parameters $\Theta$. This case does not give rise to any complications and can in general be dealt with by redefining $Z_{1}$ and $\Theta$. The other three types of changes have different implications for the statistical reliability of the model, and they concern the long-run parameters $\alpha$ and $\beta$ (suitably normalised) as well as the rank of the matrix $\Pi=\alpha \beta$.

Among others, Hansen and Johansen (1999) suggest graphical procedures to evaluate the constancy of the long-run parameters of the model. They look at the time paths of the recursively estimated eigenvalues and argue that by inspecting them it is possible to identify the breakpoints. Furthermore, they show that fluctuation tests like the one by Ploberger, Kramer and Kontrus (1989) and the LM-test by Nyblom (1989) can be applied to test the constancy of long-run parameters in cointegrated VARs. Seo (1998) defines LM tests statistics for structural changes in both the cointegrating vector and the vector of adjustment parameters for both the cases of a known and unknown breakpoint. Using Monte Carlo methods he finds that the tests for structural change of the cointegrating vector have a non-standard distribution that is equal to the one found by Hansen (1992) using the FM technique. The tests on the vector of loading weights (the adjustment coefficients) are found to have the same asymptotic distribution for models with stationary variables. ${ }^{1}$

Consider again the cointegrated model in error correction form which this time incorporates time varying $\alpha$ and $\beta$.

$$
Z_{0 t}=\Theta Z_{1 t}+\alpha(t) \beta(t)^{\prime} Z_{2 t}+\varepsilon_{t},
$$

\footnotetext{
${ }^{1}$ Similar results are obtained by Andrews and Ploberger (1994) for the test on the matrix of loading weights, but they find a different (even though still non-standard) distribution for the tests for constancy of the parameters in cointegrating relations.
} 
Within this framework there are three possible types of structural change in the model:
A) $\quad \alpha(t) \beta(t)^{\prime}=\left(\alpha \beta_{1} I_{1 t}+\alpha \beta_{2} I_{2 t}+\ldots . .+\alpha \beta_{k} I_{k t}\right)$
B) $\quad \alpha(t) \beta(t)^{\prime}=\left(\alpha_{1} \beta I_{1 t}+\alpha_{2} \beta I_{2 t}+\ldots . .+\alpha_{k} \beta I_{k t}\right)$
C) $\quad \alpha(t) \beta(t)^{\prime}=\left(\alpha_{1} \beta_{1} I_{1 t}+\alpha_{2} \beta_{2} I_{2 t}+\ldots . .+\alpha_{k} \beta_{k} I_{k t}\right)$

where $I_{j t}(j=1, \ldots, k)$ are indicators.

The first case amounts to a shift in the cointegrating coefficients. This may take the form of a shift in the parameter attached to one or more variables, and therefore a change in the cointegrating space, but may also take the extreme form of a breakdown of the long-run relationship. Case B is that of a structural break in the matrix of adjustment coefficients, and implies a change in the long-run causal structure of the model but not in the cointegrating space. A relevant example would be an attempt on the part of the monetary authorities to change the structure of the economy using specific policies. Case $\mathrm{C}$ involves a simultaneous shift in both the cointegrating and the adjustment coefficients.

The key feature to note here is that almost all the work cited above has focused on analysing structural change of the form given by model A, rather than model B. Yet we believe structural change of the form given by B is both more empirically relevant and more interesting from a policy perspective. However, we have very little insight into how such change will affect our estimation techniques, how to detect it or what its small sample implications are.

In this paper we concentrate on such cases and investigate, using Monte Carlo methods, the performance and the distribution of the full sample FIML estimates for the $\alpha$ s when there is a break in these coefficients. We will compare the performance of recursive as well as rolling FIML techniques for detecting such changes occurring at different points in time and in samples of different size. As in Hansen and Johansen (1999), we will regard the recursive and rolling estimation techniques as misspecification tests for the detection of possible instabilities in the adjustment coefficients and of the breakpoints. 
Our Monte Carlo evidence shows that in the case of a break in the as, recursive techniques like the one of Hansen and Johansen (1999) that rely upon the inspection of recursive eigenvalues are not very useful, as they tend to highlight any sort of problems that may be present in the system. Also, as the null hypothesis is that no break occurs, the inspection of the recursively-estimated coefficients may prove useful in showing their non-constancy, but still it will not be accurate enough to detect the breakpoints in individual replications.

Rolling techniques perform relatively better in that they detect the non-constancy of the parameters, providing also estimates of the parameter value after the break. However, they are not entirely satisfactory, since they only detect the region where the break is likely to occur, and they are too sensitive to the size of the rolling window and the magnitude of the break. To overcome such a problem we try two alternative approaches, finding that they are more efficient in detecting the breakpoints as well as providing the true value of the parameters of interest after the break has occurred.

The first testing procedure consists in incorporating into the system in error correction form a sequentially switching dummy for the speed of adjustment parameter, defined so that it becomes "more" statistically significant when it corresponds exactly to the break in the data. We will derive the critical values for the null of no break for different sample sizes and will examine the statistical properties of the test. We will see that such a technique has relatively high power, even in the case of a small break.We will then compare its performance to that of a time-varying version of the Kalman filter for the estimation of the parameters of interest, this being a well-established estimation methodology for detecting instabilities of parameters and breakpoints. Note also that the properties of the Kalman filter are such that it can be used in the case of multiple breaks or gradually changing coefficients.

The layout of the paper is the following: In the next section we will introduce the DGPs used for the Monte Carlo experiment. Section 3 and 4 present a sequential test for structural breaks in the causality of cointegrated VARs and the Kalman filter, as they will 
be used in our experiment. The results are discussed in section 5. A brief summary and final remarks conclude.

\section{The Monte Carlo experiment}

We have designed the DGPs according to two simple criteria: model dimension and breakpoint of the causal structure. For better management of the experiment and in order to concentrate only on the long-run causal structure of the models, we have assumed constant cointegrating rank and absence of breaks in the cointegrating coefficients. We have generated a basic cointegrated bivariate error correction model with dynamics that obey a simple autoregressive process of order one (AR(1)), and have examined the performance of the procedures under investigation allowing for the possibility of three different points in time where the causal structure changes ${ }^{2}$. The simulated model is of the following form:

$$
\Delta X_{t}=\mu+\Gamma \Delta X_{t-1}+\Pi X_{t-1}+e_{t}
$$

that under cointegration can be rewritten as

$$
\Delta X_{t}=\mu+\Gamma \Delta X_{t-1}+\alpha \beta^{\prime} X_{t-1}+e_{t}
$$

In particular we consider the case where

2.3

$$
\mu=\left[\begin{array}{l}
0 \\
0
\end{array}\right], \Gamma=\left[\begin{array}{cc}
0.3 & 0 \\
0 & 0.3
\end{array}\right], \quad \alpha=\left[\begin{array}{c}
-0.25 \\
0
\end{array}\right], \quad \beta=\left[\begin{array}{c}
1 \\
-2
\end{array}\right]
$$

and allow the matrix $\alpha$ to switch to

\footnotetext{
${ }^{2}$ Note that while a bivariate VECM can be useful to analyse several economic issues (i.e. interest rates parity, Present Value models), the simulated DGP is obviously a simple one and does not allow for generalisations of the results outside the context of the model itself.
} 


$$
\alpha^{*}=\left[\begin{array}{c}
-0.25 \\
0.125
\end{array}\right]
$$

implying that the matrix $\Pi=\alpha \beta^{\prime}$ will switch to $\Pi^{*}=\alpha^{*} \beta^{\prime}$ as follows:

$$
\Pi=\left[\begin{array}{cc}
-0.25 & 0.50 \\
0 & 0
\end{array}\right], \quad \Pi^{*}=\left[\begin{array}{cc}
-0.25 & 0.50 \\
0.125 & -0.25
\end{array}\right]
$$

The reason for considering bivariate processes is that they are relatively easy to work with and are frequently used in empirical research (e.g. Purchasing Power Parity, Uncovered Interest Parity), while higher dimensional processes would create problems of experiment management and interpretation ${ }^{3}$. In each of these processes we have considered the simple case of a unique break, consisting of a zero loading weight switching to a non-zero value at some point in time ${ }^{4}$. For the FIML-based analysis and the Kalman filter we have generated 1000 samples of size $\mathrm{T}+50$, always discarding the first 50 observations ${ }^{5}$. The data generating processes (DGPs) differ from each other in the points at which the break occurs. These are $1 / 4,1 / 2$, and $3 / 4$ of the samples. We will henceforth identify these three basic DGPs as DGP1, DGP2, and DGP3 respectively. For each of the DGPs, four sample sizes were considered, that is $\mathrm{T}=50,100,200$, and 400 .

\footnotetext{
${ }^{3}$ Toda and Phillips (1993a,1993b) have demonstrated that, unless so-called sufficient cointegration rank conditions are met, the usual $\chi^{2}$ statistics for weak exogeneity (long-run causality) for the matrix $\alpha$ may be invalid, thus invalidating any causal inference. However, we assume that these conditions are met here as we simulate a model with a stable cointegrating rank. The practitioner should verify that conditions for sufficient cointegration are met before inferring the causal structure of the model.

${ }^{4}$ Notice that, in general an unmodelled change in the parameters of a regression model may have some effect on the residuals. In our simulated DGPs this might or might not necessarily be the case, as, for example, residual autocorrelation is mostly associated to changes in the deterministic terms (see, e.g., Perron 1989), which, however, are not considered here. We assume that only a relatively small change in one of the adjustment coefficients has occurred. The more likely problem that this causes is that full sample estimates of the coefficient in question will be a weighted average of the value of the coefficient in the two regimes, without necessarily leaving evident traces on the residuals.

${ }^{5}$ Notice that discarding the first 50 observations has the implication that the starting value of our simulated random walks will be the sum of 50 iid random variables and therefore will not be zero anymore. However, this should not constitute a problem as in the OLS and FIML estimations we have always included an intercept term to account for the non-zero intercepts.
} 
To derive the critical values and the statistical properties of the sequential test we have carried out 2500 replications.

In the FIML analysis we will perform various types of estimation. Specifically, we will first estimate full sample models performing the usual Johansen $(1988,1991)$ cointegration test without checking the chosen of model for misspecification, obtaining the estimates of the parameters of interest in the models. The objective is to investigate the statistical properties of the FIML estimates for the loading weights in the presence of a unique and unmodelled permanent break for different sample sizes and breakpoints. We will then re-estimate the model recursively to investigate whether the FIML technique is able to detect the presence of the breaks via the inspection of the recursive eigenvalues and the recursive estimates. Furthermore, we will estimate the loading weights from the model in error correction form using OLS, where the cointegrating relation is the one obtained recursively using FIML, and we will investigate their statistical significance in all the recursions for all the sample sizes. Lastly, we will perform a rolling estimation of the cointegrating coefficients and the adjustment parameters, infer the statistical properties of the FIML estimator in small samples, and investigate the possibility of detecting the break in the DGPs using such a technique. In our experiments, the initial sample size for the recursive estimation is 20 . For the rolling estimation the sizes of the moving window are 30,40 and 50 data points.

Next, we will use an OLS-based sequential test that is based on defining a sequentially switching dummy for the loading weights in order to detect the occurrence of a break and the breakpoint itself. We will also report the results obtained on the same DGPs using a time-varying version of the Kalman filter. The latter will be used as a benchmark to compare the relative performance of the sequential-test- and the FIML-based procedures in the case of a shift in the causality between variables in the model. These two last techniques are described in the following sections.

All the simulations and the estimation have been carried out using codes written in GAUSS. 


\section{A sequential test for structural change}

Consider the following cointegrating VAR in error correction form,

$$
\Delta X_{t}=\mu+\Gamma_{1} \Delta X_{t-1}+\ldots+\Gamma_{k-1} \Delta X_{t-k+1}+\alpha\left(\beta^{\prime} X_{t-k}\right)+e_{t}
$$

where $\beta^{\prime} \mathrm{X}_{\mathrm{t}-\mathrm{k}}$ is the stationary linear combination of the non-stationary levels that is assumed to be stable over time, and $\alpha$ represents the speed at which the variables in $\left\{\mathrm{X}_{\mathrm{t}}\right\}$ adjust to disequilibrium. We consider the case where the matrix $\alpha$ switches over time to some matrix $\alpha^{1}$. As an example consider the simple bivariate case where

$$
\alpha=\left[\begin{array}{c}
\alpha_{11} \\
0
\end{array}\right], \quad \alpha^{1}=\left[\begin{array}{c}
\alpha_{11}^{1} \\
\alpha_{21}^{1}
\end{array}\right], \quad \quad \alpha_{21}^{1} \neq 0
$$

Such a switching may be caused by many phenomena. Consider the case of the UK joining the exchange rate mechanism (ERM) at some point in time. Suppose that we are estimating a simple model of uncovered interest parity between the rates of Germany and UK. Assuming that the two rates are cointegrated before and after the entry of the UK into the ERM, it is reasonable to expect that, from the date at which the UK joins the ERM, its rates start being affected by the German ones. In this case, in the UK equation, the coefficient of the speed of adjustment towards equilibrium (in our example $\alpha_{21}$ ) would switch from a zero to a non-zero value.

The testing procedure consists in estimating such a model including a dummy that should be multiplied by the error correction term. This dummy variable is then switched on (sequentially) for sub-samples of the data. The strategy consists in observing for which sub-period this switching dummy is statistically "more" significant.

The estimated model will be: 


$$
\begin{aligned}
& \Delta X_{t}=\mu+\Gamma_{1} \Delta X_{t-1}+\ldots+\Gamma_{k-1} \Delta X_{t-k+1}+\alpha\left(\beta^{\prime} X_{t-k}\right)+\alpha^{*} D_{t}\left(\beta^{\prime} X_{t-1}\right)+e_{t} \\
& D_{t}=I(t \geq \delta)
\end{aligned}
$$

where $\delta_{0} \leq \delta \leq \mathrm{T}-\delta_{0}$. In our case we take $\delta_{0}=2$ and $\mathrm{T}$ is the sample size. For fixed and identified $\beta$, to facilitate our task we can estimate the VECM equation by equation, so as to focus on one row of $\alpha$ at a time.

For each of the $\alpha_{i}$, we consider the test statistics for the null hypothesis of no-break of the form

$$
\tau=\max _{\delta \in D}\left|t_{\delta}\right|
$$

where $t_{\delta}$ is the t-statistic associated with the dummy $\mathrm{D}_{t}$. As there are no tabulated critical values for such a test statistics, we had to derive the $90 \%$ and $95 \%$ critical values for the null of no break for sample sizes of 50, 100, 200, 400, which are displayed in Table $4 .{ }^{6}$ It is clear that this testing technique can only be used to detect a single permanent shift in the coefficient to which it is attached. By contrast, when the prior is that there are multiple breaks and when the breakpoints are in general unknown, it could be preferable to use a time-varying technique such as the Kalman filter outlined below.

\section{$4 \quad$ Kalman filtering}

The Kalman $(1960,1963)$ filter technique is adopted to estimate linear models with timevarying coefficients ${ }^{7}$. This class of models consists of two equations: the transition equation, describing the evolution of the state variables, and the measurement equation, describing how the observed data are generated from the state variables. This approach is extremely useful for investigating the issue of parameter constancy, because it is an updating method producing estimates for each time period based on the observations

\footnotetext{
${ }^{6}$ It is worth highlighting that the distribution of $\tau$ depends among other things on the value of the startup parameter $\delta_{0}$. This dependence however, should disappear as T becomes larger.
}

7 For a more detailed account of Kalman filtering, see Cuthbertson et al (1992). 
available up to the current period. It is important to realise that recursive OLS estimation (or moving window OLS estimation) is not a suitable technique to use here. Recursive estimation is essentially a test of structural stability. We can set up as the null the hypothesis that the parameters are constant and see if it can be rejected through recursive estimation. But as the underlying assumption of OLS is always that the parameters are constant, recursive estimation does not provide a consistent estimate of a time-varying parameter.

Let the Kalman Filter measurement equation be:

$4.1 \quad y_{t}=x_{t} \theta_{t}+\varepsilon_{t} \quad \varepsilon_{\mathrm{t}} \sim N\left(0, H_{t}\right)$

and the transition equation be:

4.2 $\theta_{t}=F \theta_{t-1}+\eta_{t} \quad \eta \sim \mathrm{N}\left(0, \mathrm{Q}_{\mathrm{t}}\right)$

with the initial conditions given by:

$4.3 \quad \theta_{0} \sim N\left(\theta_{0}, \sigma^{2} P_{0}\right)$

When $\mathrm{F}=\mathrm{I}$ and $\mathrm{Q}_{\mathrm{t}}=0$, the model is reduced to the standard normal OLS regression model. The matrices $F, H_{t}$ and $Q_{t}$ are assumed to be known, and the problem is obtaining estimates of $\theta_{t}$ using information $I_{t}$ available up to time t. The process of evaluating the conditional expectation of $\theta_{t}$ given $I_{t}$ is known as filtering. The evaluation of $\theta_{t}$ given $I_{s}$, with $s>t$, is instead referred to as smoothing, whereas the estimation of $\theta_{t}$ with $s<t$ is called prediction. Kalman (1960) derived the basic results to obtain filtered and smoothed estimates of $\theta_{\mathrm{t}}$ recursively. The prediction equation is given by:

$$
\hat{\theta}_{t / t-1}=F \hat{\theta}_{t-1}
$$


and the covariance matrix is defined as:

$$
P_{t / t-1}=F P_{t-1} F^{\prime}+Q_{t}
$$

Finally, the updating formulae are given by:

$$
\hat{\theta}_{t}=\hat{\theta}_{t / t-1}+P_{t / t-1} x\left(y_{t}-x^{\prime} \hat{\theta}_{t / t-1}\right)\left(x^{\prime} P_{t / t-1} x+H_{t}\right)
$$

and

$$
P_{t}=P_{t / t-1}-P_{t / t-1} x^{\prime} x P_{t / t-1}^{\prime} /\left(x^{\prime} P_{t / t-1} x+H_{t}\right)
$$

Before starting the estimation process, one has to specify the vector of prior coefficients $\theta_{\mathrm{t}}$ and the matrix $\mathrm{Q}_{\mathrm{t}}$. By estimating the long-run relationship in this way one obtains a vector containing the evolving state coefficients which show whether the relative importance of the factors driving the dependent variable has changed over time.

In our case we start from a model in error correction form such as:

$$
\Delta X_{t}=\mu+\Gamma_{1} \Delta X_{t-1}+\ldots+\Gamma_{k-1} \Delta X_{t-k+1}+\alpha_{t} \beta^{\prime} X_{t-k}+e_{t}
$$

where as usual $e_{t}$ are assumed to be iid $\mathrm{N}(0, \Sigma)$, assume the $\Gamma_{\mathrm{i}}$ and $\beta$ are not time-varying, and estimate equation by equation each of the adjustment coefficients contained in the matrix $\alpha_{t}$ with the Kalman filter (so that $\theta$ in equation 4.1 and above is equal to $\alpha$ here), under the assumption that each of these coefficients follows a random walk process such that

$$
\alpha_{t}=\alpha_{t-1}+v_{t}, \quad v_{t} \text { is } \mathrm{N}\left(0, \sigma^{2} I\right)
$$


Using this technique we will obtain the time path of the adjustment coefficients for all the DGPs considered. This should reveal changes in the values of the adjustment coefficients as well as the unknown breakpoints.

\section{$5 \quad$ Results}

We start with the FIML estimation using the whole sample. The first result concerns the estimates of the cointegrating coefficients denoted as $\beta_{1}$ and $\beta_{2}$ respectively in Table 1. These coefficients (standardised with respect to $\beta_{1}$ ) are always estimated accurately by the FIML-based procedure in all the DGPs and for all the sample sizes. This indicates that, in the case under investigation, the presence of a break in the adjustment coefficients does not seem to affect the cointegration test or the estimation of the matrix of cointegrating coefficients. As expected, the estimates of $\alpha_{2}$ are a weighted average of the two values that $\alpha_{2}$ takes before and after the break. These are never too close to 0.125 , that is the value that $\alpha_{2}$ takes after the break, and in some cases (mostly in DGP3) they reach numerical values close to zero.

When it comes to accounting for breaks ${ }^{8}$ in the long-run causality structure of a system, the Johansen procedure fails to do so for breaks in the three DGPs in the case of smaller samples $(\mathrm{T}=50)$. In the case of the samples of size 100 it fails to account for the breaks in DGP2 and DGP3. In the case of DGP1 the hypothesis of a coefficient that is significantly different from zero would not be rejected. For the samples of larger dimensions (200 and 400) the results are different depending on the point in time at which the break occurs.

\begin{tabular}{|c|c|c|c|c|c|c|}
\hline & $D G P 1$ & & $D G P 2$ & & DGP3 & \\
\hline & Mean & Std. Dev. & Mean & Std. Dev. & Mean & Std. Dev. \\
\hline \multicolumn{7}{|l|}{$T=50$} \\
\hline$\alpha 1$ & -0.278 & 0.1919 & -0.2663 & 0.056 & -0.294 & 0.2117 \\
\hline$\alpha 2$ & 0.0982 & 0.1105 & 0.0633 & 0.0623 & 0.0191 & 0.117 \\
\hline$\beta 1$ & 1 & - & 1 & - & 1 & - \\
\hline$\beta 2$ & -1.9674 & 0.3113 & -2.0089 & 0.1417 & -1.9573 & 0.3775 \\
\hline$T=100$ & & & & & & \\
\hline
\end{tabular}

\footnotetext{
${ }^{8}$ By failing to account for breaks in the full sample estimation, we mean that the coefficient estimates are distributed such that it would not be possible to reject the hypothesis of a zero coefficient.
} 


\begin{tabular}{|l|cc|cc|cc|}
\cline { 2 - 7 }$\alpha 1$ & -0.2558 & 0.0375 & -0.2542 & 0.0381 & -0.2555 & 0.038 \\
$\beta 2$ & 0.0894 & 0.043 & 0.0539 & 0.0411 & 0.0259 & 0.0376 \\
$\beta 1$ & 1 & - & 1 & - & 1 & - \\
\hline $\mathbf{T}=\mathbf{2 0 0}$ & -2.0096 & 0.0913 & -2.0069 & 0.073 & -2.0035 & 0.0627 \\
\hline$\alpha 1$ & & & & & & \\
$\alpha 2$ & -0.2508 & 0.0253 & -0.2495 & 0.0252 & -0.2508 & 0.0258 \\
$\beta 1$ & 0.0852 & 1.0298 & 0.0523 & 0.0289 & 0.0248 & 0.0266 \\
$\beta 2$ & 1 & - & 1 & - & 1 & - \\
\cline { 1 - 6 } $\mathbf{T}=\mathbf{4 0 0}$ & -2.0016 & 0.0437 & -2.0036 & 0.0369 & -2.0025 & 0.0319 \\
\hline$\alpha 1$ & & & & & & \\
$\alpha 2$ & -0.2495 & 0.0182 & -0.2482 & 0.0183 & -0.2492 & 0.0184 \\
$\beta 1$ & 0.0845 & 0.0209 & 0.0515 & 0.0204 & 0.0241 & 0.0184 \\
$\beta 2$ & 1 & - & 1 & - & 1 & - \\
\hline
\end{tabular}

If the break occurs at $1 / 4$ of the sample as in DGP 1 , this is detected and the hypothesis of a zero coefficient on $\alpha_{2}$ would be easily rejected ${ }^{9}$. When the break occurs in the middle of the sample (DGP2) the results obtained are different for the two sample sizes of 200 and 400. With a sample size of 200 we would not be able to reject the hypothesis of a zero coefficient on $\alpha_{2}$ at the $95 \%$ level, while the same hypothesis would be rejected for a sample of 400, where we would find a coefficient on $\alpha_{2}$ that is significantly different from zero. In the case of DGP3 the hypothesis of a non-zero coefficient would be rejected for both the samples of 200 and 400. All these results are reported in Table $1^{10}$.

Following Hansen and Johansen (1999), we consider recursive estimates of the eigenvalues and the coefficients for the purpose of detecting any instability of the coefficients and possible breakpoints. If we examine Graphs 1 and 2 for DGP2 of size 100 it is clear that the inspection of the recursive eigenvalues does not provide any information on the occurrence of a break in the adjustment parameters. In fact, there is no change in the pattern of the eigenvalues in the graph showing the average of 1000

\footnotetext{
${ }^{9}$ Notice that the rejection of the hypothesis of a zero in favour of a non-zero coefficient would not account for the fact that the first $\mathrm{T} / 4$ values are actually zero.

${ }^{10}$ It needs to be pointed out that these poor results are largely expected. This is because in reality we are estimating a misspecified model using FIML, which instead makes precise assumptions on the residuals of the model, so that likelihood theory is invalid.
} 
replications at the points where the break occurs. The recursively estimated matrix of loading weights appears to be relatively more informative in terms of graphic analysis.

The plot of the recursive estimates of the loading weights shows their instability over time. In the graph of the time path of the recursive loading weights for DGP2 as an average of 1000 replications and a sample of size 100, we observe a clear tendency in the value to increase after the breakpoint that is identifiable by a slight kink. At first sight, we could be tempted to conclude that the recursive estimates provide excellent results in terms of the detection of the break as well as the breakpoint. However, the results obtained in single replications (not displayed) suggest that recursive analysis is useful only in the detection of instability in the parameter estimates - it cannot be relied upon for the detection of the breakpoint because the corresponding kink is not clearly observable in single replications.

In any case, the statistical ability of the recursive procedure in detecting the breaks is made doubtful by the fact that, when the $\alpha$ coefficients become significantly different from zero in statistical terms, they do so with a considerable time lag. We have obtained these results estimating recursively the loading weights as the coefficients attached to the error correction term (as obtained with recursive FIML) in a VECM using OLS. The OLS estimation and the inspection of the t-values for the $\alpha_{2}$ coefficient provide us with results that are different depending on the sample size and the breakpoint. They indicate (see Table 2) that, when $\alpha_{2}$ becomes significant, it does so with a delay that goes from 60 to 100 points depending on sample size and breakpoint.

\begin{tabular}{|l|ccccccc|}
\hline Table 2 & \multicolumn{7}{|c|}{ t-values of recursive estimates of $\boldsymbol{\alpha}_{2}$} \\
\hline DGP1 & $\mathbf{T} / \mathbf{4}$ & $(3 / 8) \mathrm{T}$ & $\mathrm{T} / 2$ & $(5 / 8) \mathrm{T}$ & $(3 / 4) \mathrm{T}$ & $(7 / 8) \mathrm{T}$ & $\mathrm{T}$ \\
\hline$T=50$ & - & 0.849822 & 1.108077 & 1.302761 & 1.493226 & 1.632482 & $1.730184^{*}$ \\
$T=100$ & 0.266668 & 0.82887 & 1.203619 & 1.548965 & $1.826124^{*}$ & $2.08068^{* *}$ & $2.34645^{* *}$ \\
$T=200$ & 0.193132 & 0.957559 & 1.522429 & $2.010072^{* *}$ & $2.435999^{* *}$ & $2.822665^{* *}$ & $3.17873^{* *}$ \\
$T=400$ & 0.177762 & 1.253367 & $2.083647^{* *}$ & $2.785881^{* *}$ & $3.395936^{* *}$ & $3.95377^{* *}$ & $4.46386^{* *}$ \\
\hline DGP2 & $\mathrm{T} / 4$ & $(3 / 8) \mathrm{T}$ & $\mathbf{T} / \mathbf{2}$ & $(5 / 8) \mathrm{T}$ & $(3 / 4) \mathrm{T}$ & $(7 / 8) \mathrm{T}$ & $\mathrm{T}$ \\
\hline$T=50$ & - & 0.382371 & 0.45922 & 0.687334 & 0.90886 & 1.072572 & 0.247567 \\
$T=100$ & 0.266668 & 0.236806 & 0.195866 & 0.615602 & 0.937328 & 1.225415 & 1.519585
\end{tabular}




\begin{tabular}{|l|ccccccc|}
$T=200$ & 0.193132 & 0.155975 & 0.150348 & 0.749731 & 1.232245 & $1.667304 *$ & $2.059217 * *$ \\
$T=400$ & 0.177762 & 0.152599 & 0.12635 & 0.967444 & $1.667098 *$ & $2.288966^{* *}$ & $2.853594 * *$ \\
\hline DGP3 & $\mathrm{T} / 4$ & $(3 / 8) \mathrm{T}$ & $\mathrm{T} / 2$ & $(5 / 8) \mathrm{T}$ & $\mathbf{( 3 / 4 ) \mathbf { T }}$ & $(7 / 8) \mathrm{T}$ & $\mathrm{T}$ \\
\hline$T=50$ & - & 0.382371 & 0.332199 & 0.282985 & 0.377341 & 0.553404 & 0.739095 \\
$T=100$ & 0.266668 & 0.236806 & 0.195866 & 0.156649 & 0.128573 & 0.496831 & 0.811484 \\
$T=200$ & 0.193132 & 0.155975 & 0.150348 & 0.137181 & 0.106946 & 0.628267 & 1.062327 \\
$T=400$ & 0.177762 & 0.152599 & 0.12635 & 0.111431 & 0.097988 & 0.812283 & 1.428967 \\
\hline$*$ denotes a coefficient $\alpha_{2}$ significantly different from zero at 10\% level & & & \\
$* *$ denotes a coefficient $\alpha_{2}$ significantly different from zero at 5\% level & & & & \\
$T=$ \\
The bold indicates the breakpoints in the DGPs
\end{tabular}

More in detail, we see that in samples of size $50 \alpha_{2}$ is only significant at a $90 \%$ level in DGP1. For the samples of size 100, it is significant only in DGP1 and it starts to be so only when $3 / 4$ of the sample points are included. For other sample sizes the story is much the same, with $\alpha_{2}$ that takes values significantly different from zero after 100 points have been added from the recursive procedure. Notice that the $\alpha_{2}$ coefficients are never significant in the case of DGP3 regardless of the sample size. This implies that if we had a sample of 400 points with the last 100 being different from zero, and we analysed it using recursive methods, we would not be able to detect the presence of a break at all. This result highlights a critical limit of the FIML estimation even in its recursive version.

To summarise, while the t-values on $\alpha_{2}$ could be used in some cases to confirm the presence of a break, they cannot be relied upon for the precise detection of the breakpoint or its magnitude. At any rate, recursive estimation is not very useful to account for a break in samples that are smaller than 200 and for all sample sizes when the break occurs at $3 / 4$ of the series. Furthermore, it is important to highlight that the graphs of the recursive coefficients are obtained as averages of 1000 replications, and show the breakpoints much more clearly than in the case of individual replications.

Rolling estimation provides results that are a little more informative. Indeed (see Graphs 3 and 4), if used graphically, the rolling procedure shows the occurrence of a break in the as. Unfortunately, the estimates of the alphas are not statistically significant in the case 
of the size of a rolling window of 30 . This relatively small window, and the size of the break in terms of the value to which the coefficient $\alpha_{2}$ switches to, do not allow to obtain statistically useful results. The implication is that the inspection of the rolling coefficients and relative t-values from individual repetitions is not helpful (Table 3).

\begin{tabular}{|l|ccccccc|}
\hline \multicolumn{1}{|l|}{ Table 3} & t-values of rolling estimates of $\boldsymbol{\alpha}_{2}$ \\
\hline Size of rolling window & $\mathrm{T} / 4$ & $(3 / 8) \mathrm{T}$ & $\mathrm{T} / \mathbf{2}$ & $(5 / 8) \mathrm{T}$ & $(3 / 4) \mathrm{T}$ & $(7 / 8) \mathrm{T}$ & $\mathrm{T}$ \\
\hline 30 & 0.134419 & 0.421034 & 0.827222 & 1.20794 & 1.529823 & 1.499496 & 1.505702 \\
40 & 0.358436 & 0.582036 & 0.911967 & 1.292864 & $1.64591 *$ & $1.848319 *$ & $1.862825 *$ \\
50 & 0.500187 & 0.748444 & 0.946235 & 1.265746 & 1.510874 & $1.850584 *$ & $2.130953^{* *}$ \\
\hline $\mathrm{T}=100$ & & & & & & \\
Breakpoint is at $\mathrm{T} / 2$ (in bold) \\
* denotes a coefficient significantly different from zero at the 90\% level \\
** denotes a coefficient significantly different from zero at the 95\% level \\
\hline
\end{tabular}

When we use a rolling window of 40 points we get better results, in the sense that we observe the presence of a break in the data and we are be able to obtain coefficients that are significantly different from zero at the $10 \%$ level. In order to get statistically significant results at a conventional 5\% level we should use a rolling window of at least 50 points. This implies that, in order for the break to be detectable, it has to be larger than 50 points, and that therefore we would need relatively large samples. Furthermore, the larger size of the rolling window complicates the graphic analysis in terms of the detection of the break points, as can be gathered from Graphs 3, 4 and 5. In empirical work, apart from financial series, we are rarely dealing with very large samples; given also the need for a relatively large minimum size of the break, the reliability of the technique in statistical terms is limited. Nevertheless, it seems that graphically it performs better than the recursive procedure in detecting the interval (even so this is sometimes a wide one) within which the break occurs and the value of the coefficient after the break.

The sequential test represents without doubt a better alternative to the techniques examined so far, as it performs better with respect to the accuracy with which it detects both the point and the magnitude of the structural break (in terms of the value that $\alpha_{2}$ takes after the break). In general, the test displays reasonable statistical properties. These 
are reported in Table 4. Specifically, the power of the test is always relatively high, even for samples of size 100, regardless of the location of the breakpoint (however it is higher when the break occurs in the middle of the sample). Also the test can be considered very reliable for samples of 200 points. For very large samples its precision is extreme. The loss of power in smaller samples can be justified on the grounds of its non-standard distribution and the relatively high critical values for samples of 50 data points (that are almost the same as the ones for 100). If we use the test graphically, we can see (Graph 6) that the coefficient attached to the switching dummy displays a kink that allows us to detect the breakpoint quite accurately. Also, notice that the corresponding coefficient takes the value of 0.125 , indicating accuracy in estimating the magnitude of the break. Moreover, as far as statistical significance is concerned, the coefficient attached to the dummy at the breakpoint is always highly significant at the $95 \%$ level in single replications.

\begin{tabular}{|c|c|c|c|c|c|}
\hline \multicolumn{6}{|c|}{ Table 4. Sequential test - Statistical properties based on 2500 replications } \\
\hline & \multicolumn{5}{|c|}{ Critical values } \\
\hline & Quantiles & $\mathrm{T}=50$ & $\mathrm{~T}=100$ & $\mathrm{~T}=200$ & $\mathrm{~T}=400$ \\
\hline & 0.05 & 2.93 & 2.94 & 2.98 & 3.02 \\
\hline & 0.10 & 2.61 & 2.64 & 2.69 & 2.71 \\
\hline & & \multicolumn{4}{|c|}{ Power as percentage of rejections of the null } \\
\hline \multirow[t]{2}{*}{ DGP1 } & 0.05 & 0.21 & 0.44 & 0.74 & 0.97 \\
\hline & 0.10 & 0.32 & 0.55 & 0.83 & 0.98 \\
\hline \multirow[t]{2}{*}{ DGP2 } & 0.05 & 0.31 & 0.56 & 0.88 & 1.00 \\
\hline & 0.10 & 0.42 & 0.69 & 0.98 & 1.00 \\
\hline \multirow[t]{2}{*}{ DGP3 } & 0.05 & 0.23 & 0.44 & 0.75 & 0.98 \\
\hline & 0.10 & 0.34 & 0.58 & 0.86 & 0.99 \\
\hline
\end{tabular}

The relatively good performance of the sequential test is only limited by the possible occurrence of multiple breaks. In this case the significance of the switching dummy is affected and the test is not very reliable. The Kalman filter may provide the solution to this kind of problems. In theory, the fact that we obtain the time path of the adjustment coefficients in a consistent way should enable us to detect the break quite accurately as far its timing and magnitude are concerned. The advantage of using such a technique is the possibility of detecting multiple breaks, as the Kalman filter is sensitive to all possible shifts that may occur because of its continuous updating. 
From the results obtained we can conclude that the Kalman filter may be graphically useful to detect the presence of breaks and their magnitude, and that it is also very efficient. We can see this from the included graphs (Graphs 7 and 8) that refer to coefficients estimated for samples of size 100 generated according to DGP2. Here the breakpoints and their magnitude are graphically observable, with relatively narrow margins of error, as shown by the confidence interval bands. These highlight the fact that the coefficients estimated with such a technique will always be significantly different from zero after the break has occurred, suggesting a reasonable efficiency of the Kalman. This result applies to all the DGPs and sample sizes. 


\section{Conclusions}

In this paper we have examined, using Monte Carlo methods, several alternative approaches to detecting structural changes in the adjustment coefficients of cointegrated VARs. As in Hansen and Johansen (1999), we have adopted recursive techniques as misspecification tests for the detection of breaks in the $\alpha$ coefficients and of the breakpoints. The results show that the recursive eigenvalues are not able to highlight such a type of break, whilst the recursive coefficients only show non-constancy of the parameters of interest. We have then adopted a rolling FIML-based technique for the estimation of the model. The main result is that this technique shows more convincingly the non-constancy of the $\alpha$ s and their true value after the breakpoint. However, rather than a breakpoint it detects a region where the break is likely to occur.

To overcome the drawbacks of these techniques, we used an OLS-based sequential test, which consists in incorporating a sequentially switching dummy for the speed of adjustment parameter such that it becomes more statistically significant when it corresponds to the break in the data. For this test we derived the critical values for different sample sizes and studied its statistical features. We found that the test has reasonable power at the $90 \%$ level even for relatively small samples (e.g. 100). Also, it is relatively efficient graphically, as it shows a kink at the breakpoint. Furthermore, at that point, the numerical value of the coefficient of the sequential dummy corresponds to the true value of the parameter after the break has occurred. The only limit to this technique is provided by the occurrence of multiple breaks. A possible solution to these cases is the use of the Kalman filter. We therefore have tested the performance of a time-varying version of the Kalman filter on the same DGPs, showing the graphic usefulness of such a technique for detecting breakpoints and the magnitude of the breaks. Regardless of the location of the breakpoints, this well established technique turns out to be extremely useful for both purposes. 


\section{References}

Andrews, D. W. K. (1993). "Tests for parameter instability and structural change with unknown change point." Econometrica, 61: 821-856.

Andrews D.W.K. and W. Ploberger (1994) "Optimal tests when a nuisance parameter is present only under the alternative", Econometrica, 62, 1383-1414

Banerjee, A., Lumsdaine R. L. and J. H. Stock (1992) "Recursive and sequential tests of the unit-root and trend break hypotheses: theory and international evidence", Journal of Business and Economic Statistics, 10, 271-287

Cuthbertson, K., Hall, S.G., and M.P. Taylor (1992), Applied Econometric Techniques, Harvester Wheatsheaf.

Gregory, A. W., Nason, J. M. and D. Watt (1996) "Testing for structural breaks in cointegrated relationships", Journal of Econometrics, 71, 324-341

Gregory, A. and B. E. Hansen, (1996) "Residual -based tests for cointegration in models with regime shifts", Journal of Econometrics, 70, 99-126

Hansen B. E., (1992) “Tests for parameter instability in regressions with I(1) processes", Journal of Business and Economic Statistics, 10, 321-335

Hansen H. and S. Johansen (1999) "Some tests for parameter constancy in cointegrated VAR models”, Econometrics Journal, 2, 306-333

Hao, K. and B. Inder (1996) "Diagnostic test for structural change in cointegrated regression models", Economics Letters, 50,179-187

Johansen, S. (1988), "Statistical analysis of cointegration vectors", Journal of Economic Dynamics and Control, 12, 231-254

Johansen, S. (1991), "Estimation and hypothesis testing of cointegration vectors in Gaussian vector autoregressive models", Econometrica, 59, 1551-1580.

Kalman, R.E. (1960), "A new approach to linear filtering and prediction problems", Journal of Basic Engineering, Transactions of the ASME, 82, 35-45.

Kalman, R.E. (1963), "New methods in Wiener filtering theory", in J.L. Bogdanoff and F. Kozin (eds), Proceedings of the First Symposium of Engineering Applications of Random Function Theory and Probability, 270-288, John Wiley \& Sons Inc., New York.

Lucas, R. E. (1988) “ Money demand in the United States: a quantitative review", Carnegie-Rochester Conference Series on Public Policy, 29, 137-168. 
Lumsdaine R. L. and D. H. Papell (1997) "Multiple trend breaks and the unit root hypothesis", Review of Economics and Statistics, 79, 212-218

Nyblom J., (1989) "Testing for the constancy of parameters over time", Journal of the American Statistical Association, 84, 223-230

Perron, P. (1989) "The great crash, the oil-price shock and the unit root hypothesis", Econometrica, 57, 1361-1401

Ploberger, W., Kramer, W. and K. Kontrus (1989) "A new test for structural stability in the linear regression model”, Journal of Econometrics, 40, 307-318

Quintos, C. E., (1995) "Sustainability of the deficit process with structural shifts", Journal of Business and Economic Statistics, 13, 409-417

Quintos, C. E. and P. C. B. Phillips (1993) "Parameter constancy in cointegrating regressions", Empirical Economics, 18, 675-706

Seo, B. (1998) "Tests for structural change in cointegrated systems" Econometric Theory, $14,222-259$

Stock, J. and M. Watson (1993) "A simple estimator of cointegrating vectors in higher order integrated systems”, Econometrica. 61, 783-820

Toda, H., and Phillips, P. C. B. (1993a). "The Spurious effect of unit roots on vector autoregressions: An analytical study." Journal of Econometrics, 59: 229-255.

Toda, H., and Phillips, P. C. B. (1993b). "Vector autoregressions and causality." Econometrica, 61: 1367-1394.

Zivot, E. and D.W.K. Andrews (1992) "Further evidence on the great crash, the oil-price shock and he unit root hypothesis", Journal of Business and Economic Statistics, 10, 251270 
graph 1. Recursive eigenvalues for DGP 2 of size 100 (average of 1000 replications)

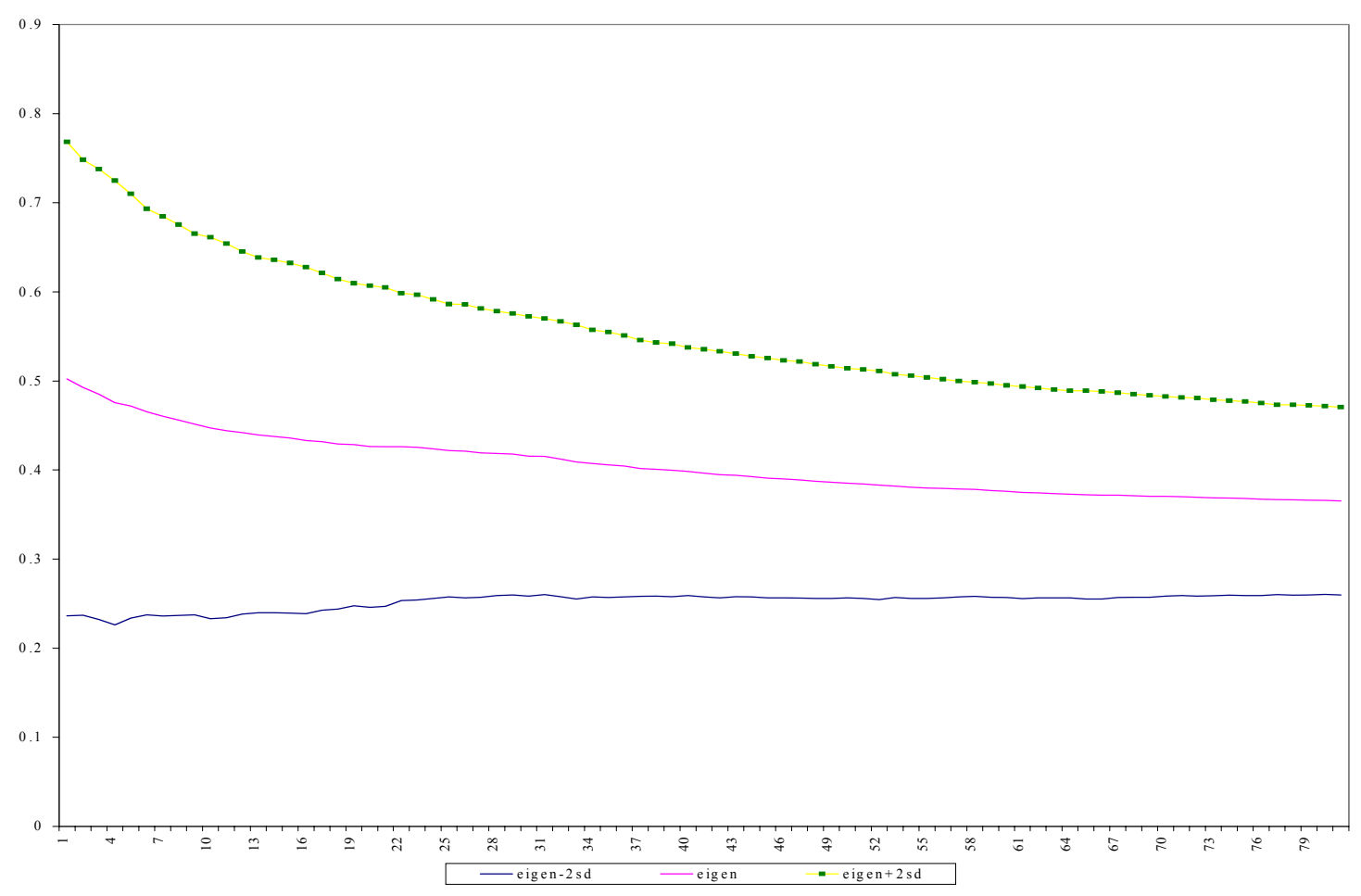

graph 2. Recursive estimates of alpha2 for DGP2 of size 100 (average of 1000 replications)

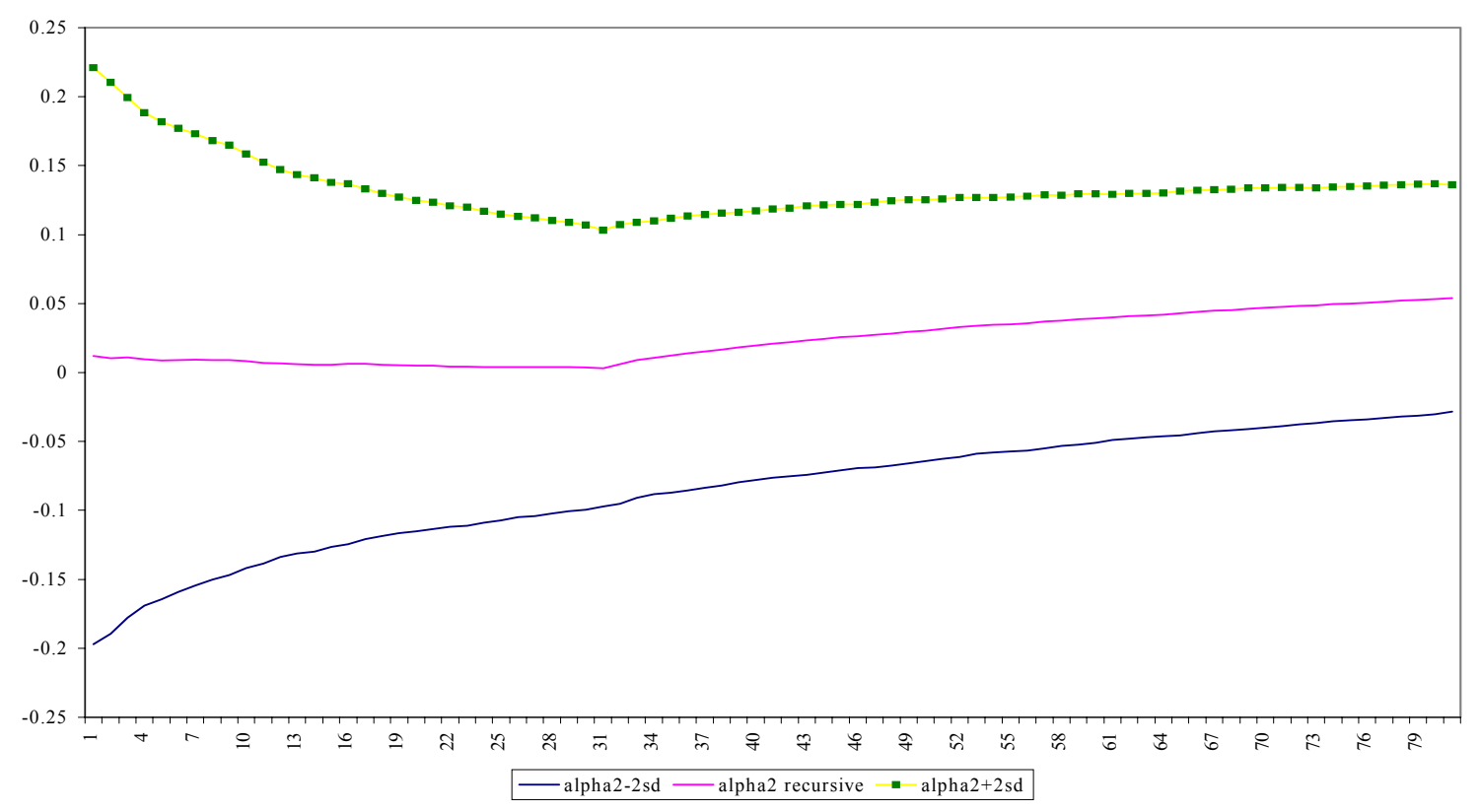




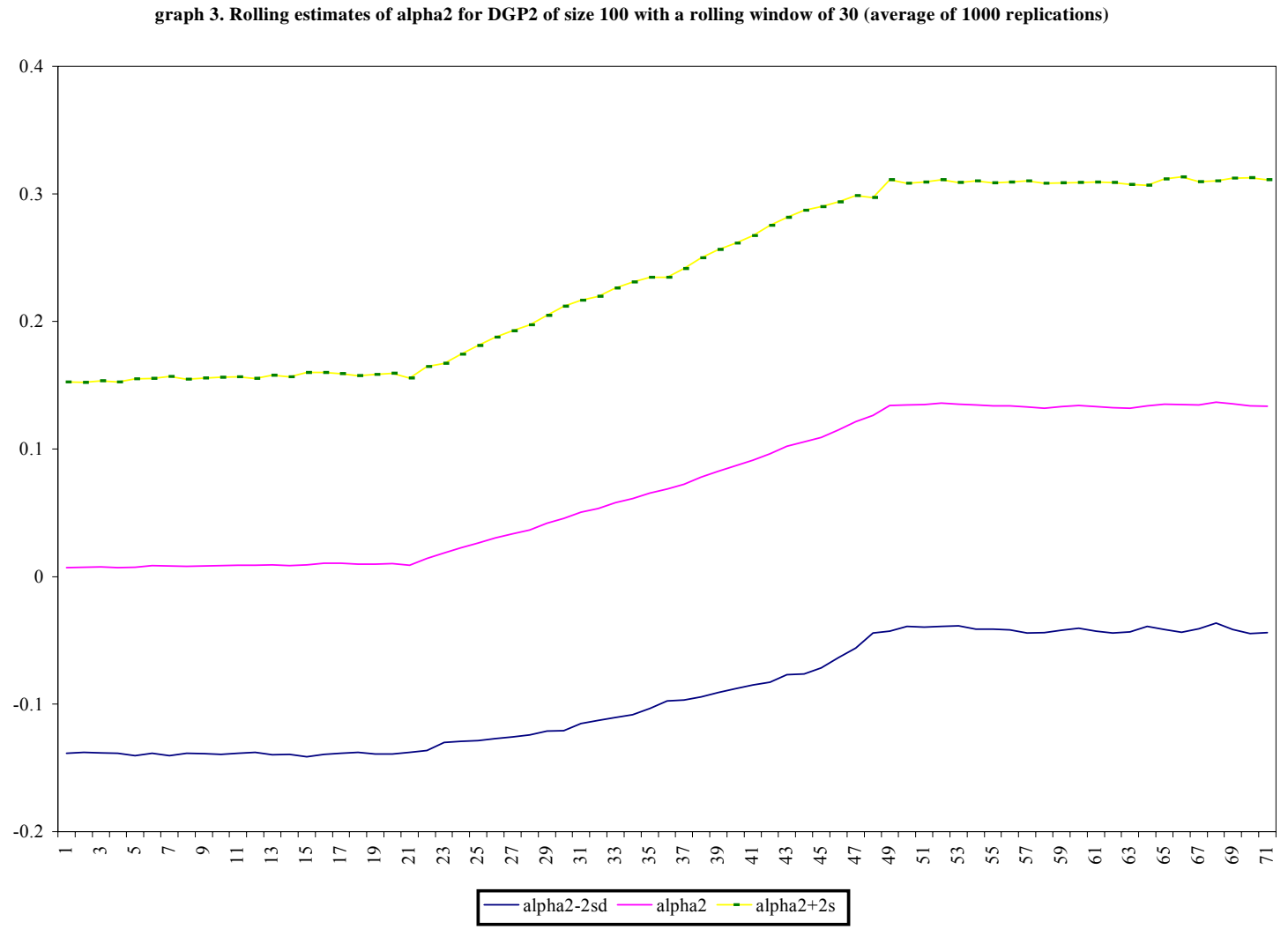

graph 4. Rolling estimates of alpha2 for DGP2 of size 100 with rolling window of 40 (average of 1000 replications)

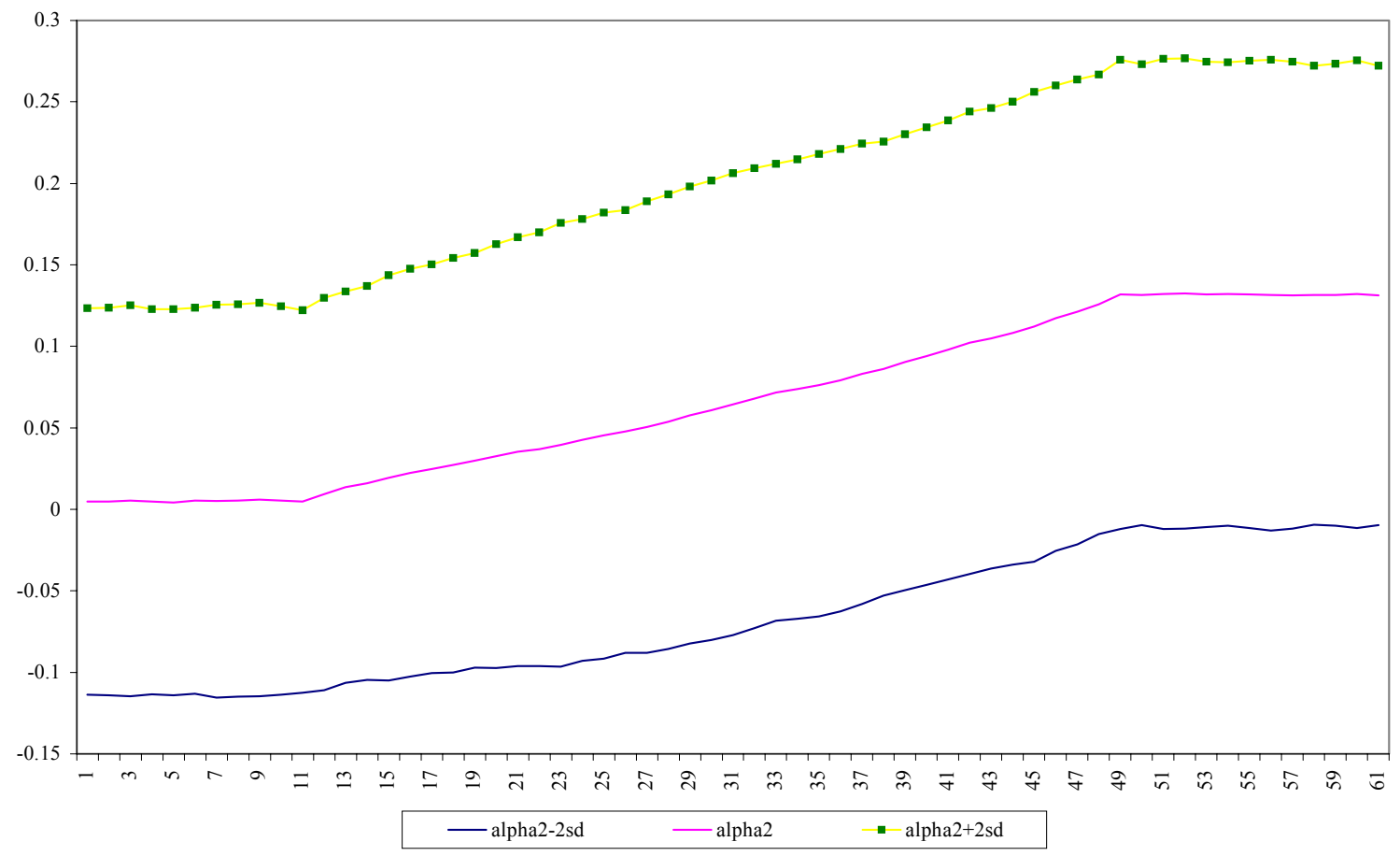


graph 5. Rolling estimates of alpha2 for DGP2 of size 100 with rolling window of 50 (average of 1000 replications)

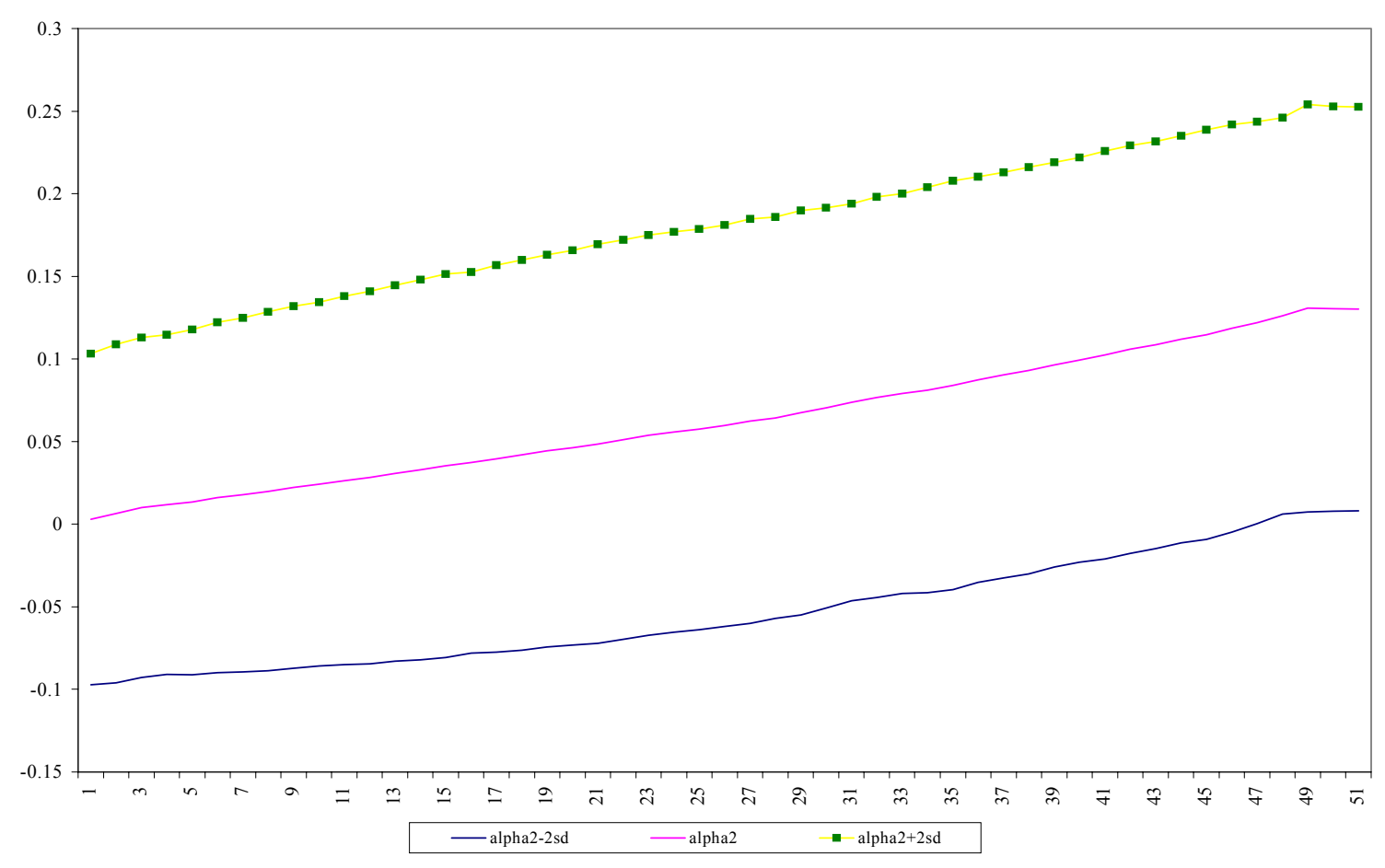

graph 6. Coefficient of sequential dumm y for DGP 2 of size 100 (average of 2500 replications)

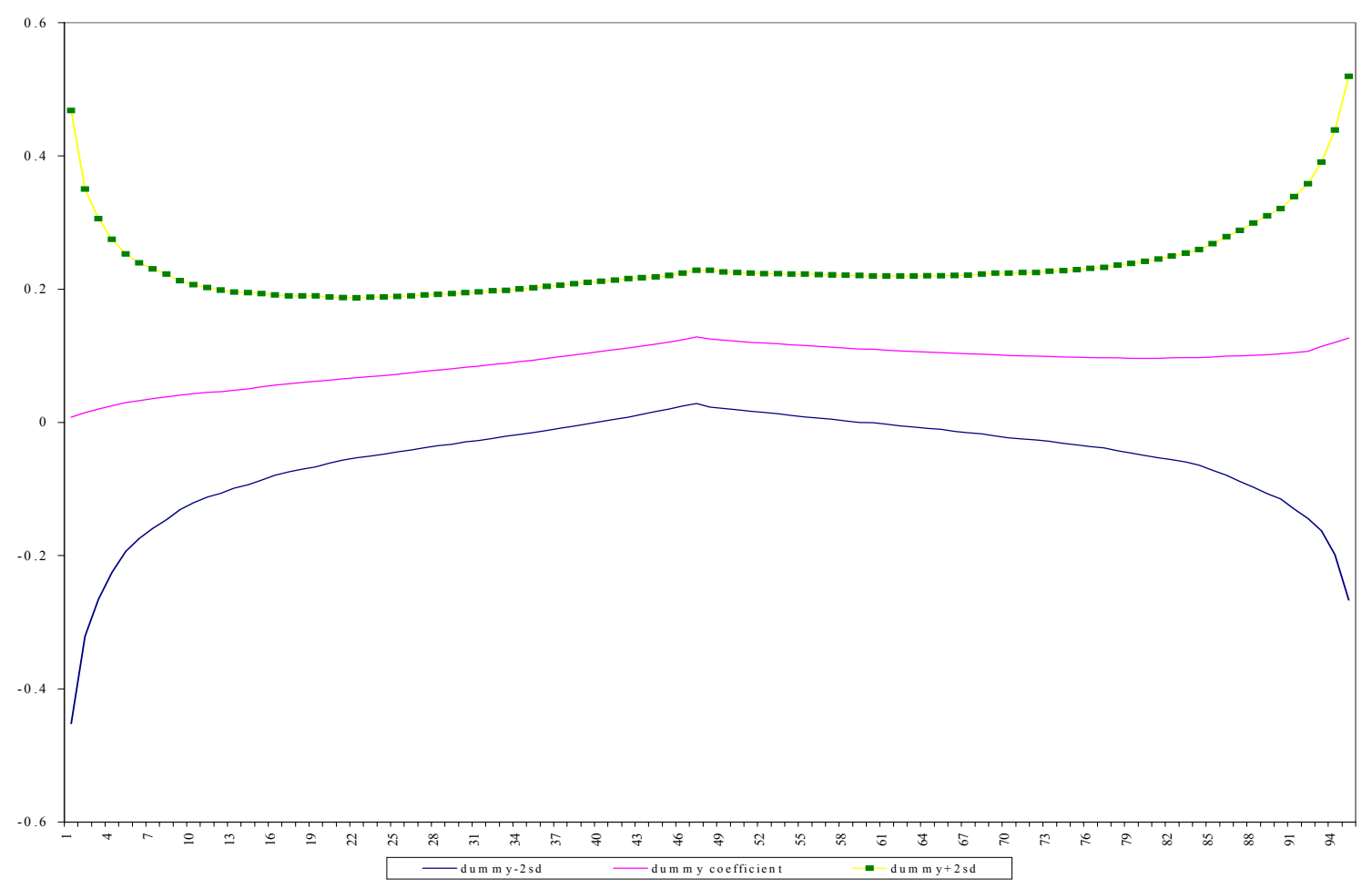


graph 7. One step ahead Kalman Filter predictions of alpha2 for DGP2 of size 100 (average of 1000 replications)

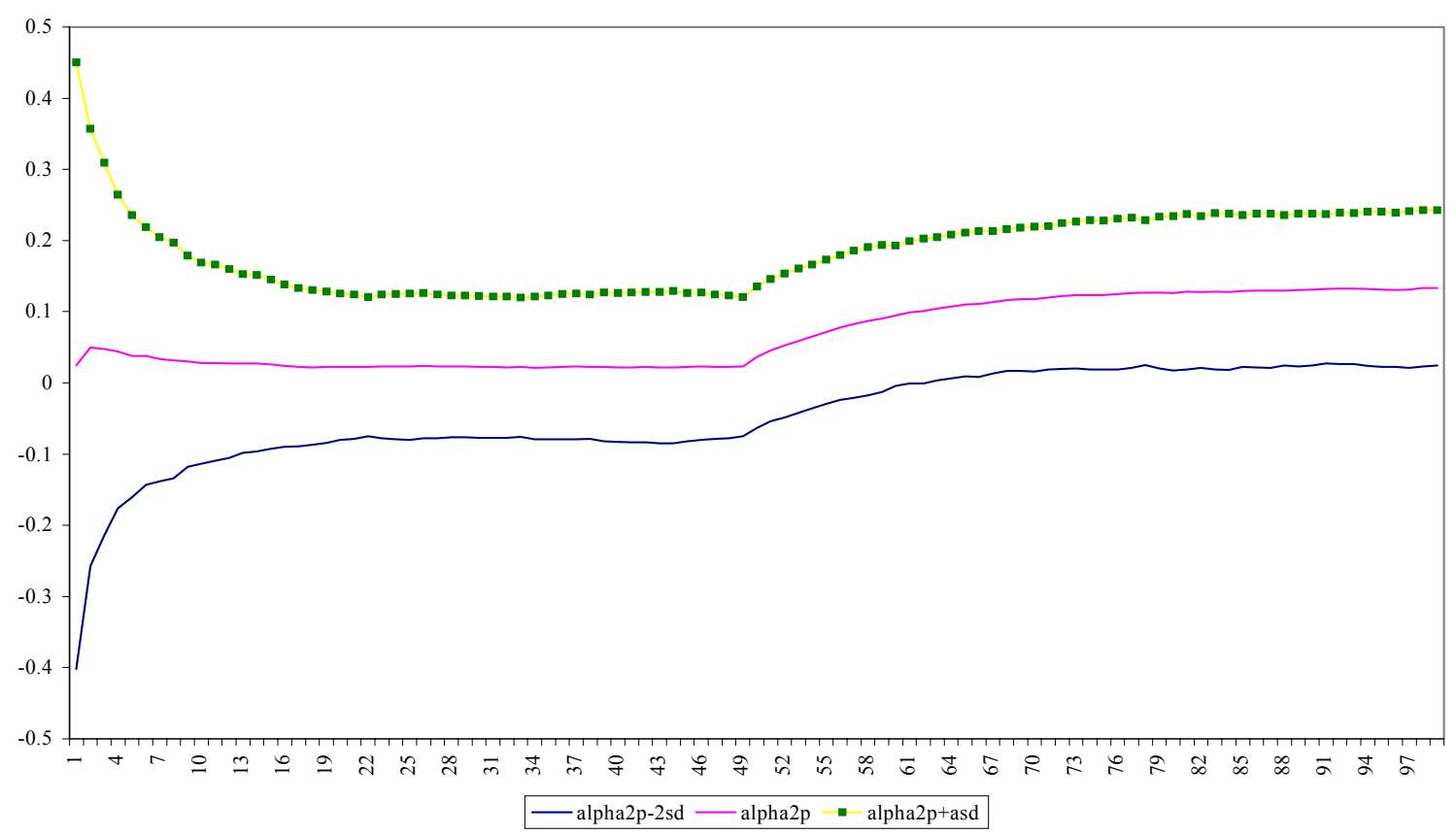

graph 8. Smoothed kalman Filter estimates of alpha 2 for DGP 2 of size 100 (average of 1000 replications)

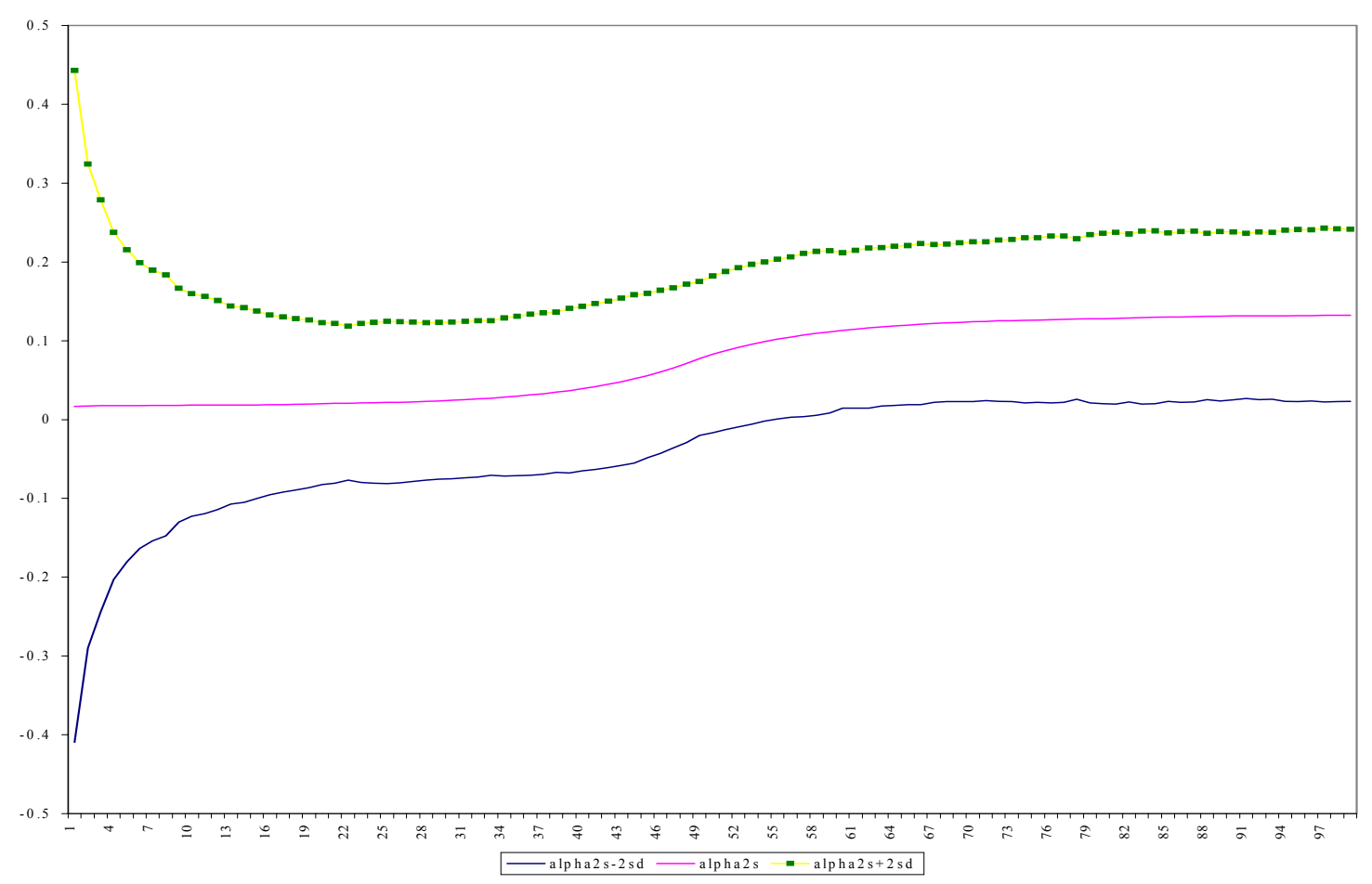

